\title{
Alternative polarisation retrieval for SCIAMACHY in the ultraviolet
}

\author{
L. G. Tilstra and P. Stammes \\ Royal Netherlands Meteorological Institute (KNMI), De Bilt, The Netherlands \\ Received: 9 December 2004 - Published in Atmos. Chem. Phys. Discuss.: 1 April 2005 \\ Revised: 8 June 2005 - Accepted: 12 July 2005 - Published: 9 August 2005
}

\begin{abstract}
We introduce an alternative method for the retrieval of polarisation in the ultraviolet by the satellite spectrometer SCIAMACHY. Unlike the operational polarisation retrieval algorithm, this method does not use the Polarisation Measurement Devices (PMDs) onboard SCIAMACHY, but only requires the reflectance signal. This makes the algorithm more robust and less sensitive to calibration errors caused by either improper characterisation of the instrument's response functions (key data) or degradation of the optical components.

The alternative polarisation retrieval is able to retrieve the full state of atmospheric polarisation in the wavelength range between 330 and $400 \mathrm{~nm}$, which is essentially the wavelength region covered by SCIAMACHY's PMD 1. This allows a direct comparison with the current operational product.

When we compare the alternative polarisation algorithm with the operational algorithm, we find in some cases agreement, but not in other cases. The alternative algorithm compares well with an analytical model of the polarisation of a cloud-free scene. Using the alternative algorithm the polarisation-sensitive feature in the SCIAMACHY reflectance around $350 \mathrm{~nm}$ is automatically corrected for.
\end{abstract}

\section{Introduction}

Satellite instruments for remote sensing of atmospheric composition like SCIAMACHY (Bovensmann et al., 1999), GOME (Burrows et al., 1999), and GOME-2 (Callies et al., 2000) all have a common problem. Due to a variety of optical elements, such as mirrors, gratings and prisms, the radiometric response functions of the instruments depend on the polarisation of the incoming light. A correction for this sensitivity is possible, but only in-flight because it requires

Correspondence to: L. G. Tilstra

(tilstra@knmi.nl) knowledge of the polarisation of the light. For that reason, these instruments are equipped with Polarisation Measurement Devices (PMDs) to determine the polarisation at a given number of distinct wavelengths.

This paper will present a validation study on the SCIAMACHY polarisation product in the UV. We validate the polarisation product by introducing an alternative method to determine the polarisation. This alternative method does not use the PMD measurements, only the reflectance, and can therefore be regarded as an independent source. From comparing this source to the operational polarisation product, we conclude that there are still discrepancies found in the operational product. We attribute these remaining problems to the calibration of the instrument.

The outline of the paper is as follows. In Sect. 2 we start with a short description of the SCIAMACHY satellite instrument. Section 3 introduces the concept of atmospheric polarisation and its relevance to the radiometric calibration of SCIAMACHY. Section 4 is completely devoted to the alternative polarisation retrieval we introduce in this paper. In Sect. 5 we compare the alternative retrieval with the operational polarisation retrieval. The paper ends with conclusions.

\section{Description of SCIAMACHY}

SCIAMACHY is a remote sensing spectrometer designed to record sunlight before and after it has been scattered by the Earth's atmosphere. The instrument is located onboard the Envisat satellite, which was launched on 1 March 2002. Using a series of eight spectral detectors, SCIAMACHY is able to cover virtually the entire spectrum between 240 and $2400 \mathrm{~nm}$. Radiance measurements can be performed in both nadir or limb mode (Bovensmann et al., 1999). These modes generally alternate each other and the blocks of data generated from these nadir or limb modes are called states.

(C) 2005 Author(s). This work is licensed under a Creative Commons License. 


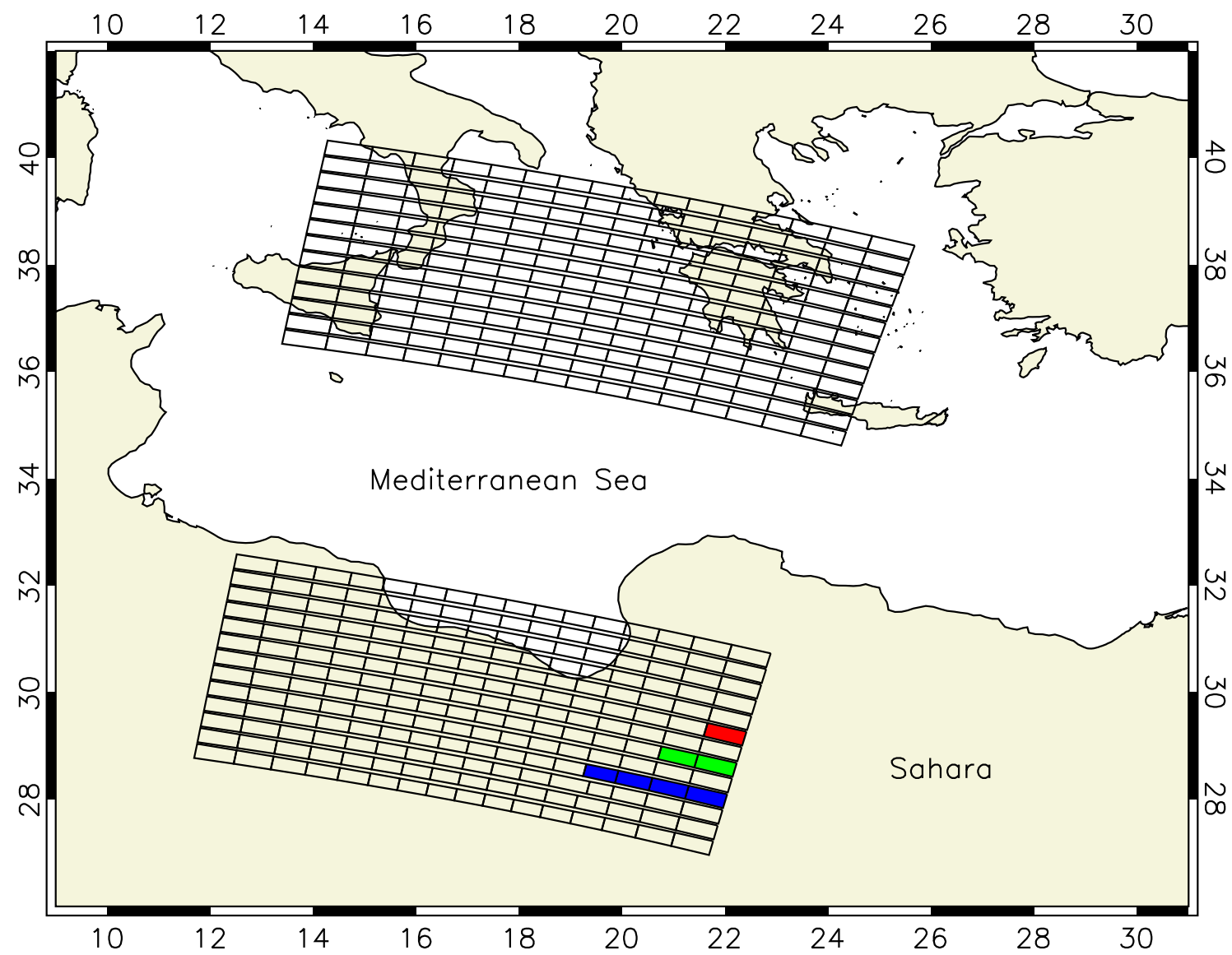

Fig. 1. Measurement approach followed by SCIAMACHY. Measurements are taken in blocks, called states. The picture shows two subsequent nadir states; the black boxes indicate the pixels of the smallest integration time $\left(0.25 \mathrm{~s}, 60 \times 30 \mathrm{~km}^{2}\right)$. The coloured regions show pixels of $0.25 \mathrm{~s}, 0.5 \mathrm{~s}$, and $1.0 \mathrm{~s}$ integration time. The data are taken from orbit 2509, dated 23 August 2002.

Figure 1 shows the footprints of two nadir states taken from orbit 2509, dated 23 August 2002. The size of a nadir state is approximately $960 \times 490 \mathrm{~km}^{2}$. The limb state that lies in between the two nadir states is not indicated. The smaller footprints are created when the instrument scans the Earth from east to west by rotating one of its internal mirrors. The resulting groundpixels are linked to an optical integration time (IT) of $0.25 \mathrm{~s}$ and their size is about $60 \times 30 \mathrm{~km}^{2}$. Indicated by the green and blue regions are pixels that are related to integration times of $0.5 \mathrm{~s}$ and $1.0 \mathrm{~s}$ that can also occur. These pixels cover roughly $120 \times 30 \mathrm{~km}^{2}$ and $240 \times 30 \mathrm{~km}^{2}$ of the Earth's surface, respectively. Next, in Fig. 2 we present a typical reflectance spectrum as measured by SCIAMACHY. The reflectance $R$ is defined as

$R=\frac{\pi I}{\mu_{0} E}$,

where $I$ is the radiance reflected by the Earth atmosphere (in $\left.\mathrm{Wm}^{-2} \mathrm{~nm}^{-1} \mathrm{sr}^{-1}\right), E$ is the solar irradiance at the top of atmosphere (TOA) perpendicular to the solar beam (in $\mathrm{Wm}^{-2}$ $\mathrm{nm}^{-1}$ ), and $\mu_{0}$ is the cosine of the solar zenith angle $\theta_{0}$. The solar irradiance is measured each day, once per 14 orbits.
The spectrum shown in Fig. 2 was taken from orbit 2509. The coloured bars on top of the graph indicate the wavelength regions that are covered by the eight spectral channels, and their overlap. Notice that the spectrum is not continuous over the full extent of the wavelength range, and that there are obvious problems in channels 7 and 8. Different parts of the spectrum are presented with different colours. This is to distinguish between wavelength regions that are read out with different integration times. For nadir observations, a spectrum generally consists of 56 of such wavelength regions, called clusters. In Fig. 2 the red, green, and blue parts of the spectrum are clusters having an integration time of $0.25 \mathrm{~s}$, $0.5 \mathrm{~s}$, and $1.0 \mathrm{~s}$, respectively.

The relationship between the radiance $I$ and irradiance $E$ on the one hand, and the raw instrument signals $S_{\text {earth }}$ and $S_{\text {sun }}$ on the other, is

$$
\begin{aligned}
& I=c_{\text {rad }} \cdot c_{\text {pol }} \cdot S_{\text {earth }} \\
& E=c_{\text {irrad }} \cdot S_{\text {sun }}
\end{aligned}
$$

Here $c_{\text {rad }}$ and $c_{\text {irrad }}$ are both calibration constants for unpolarised light that were determined pre-flight. The calibration 


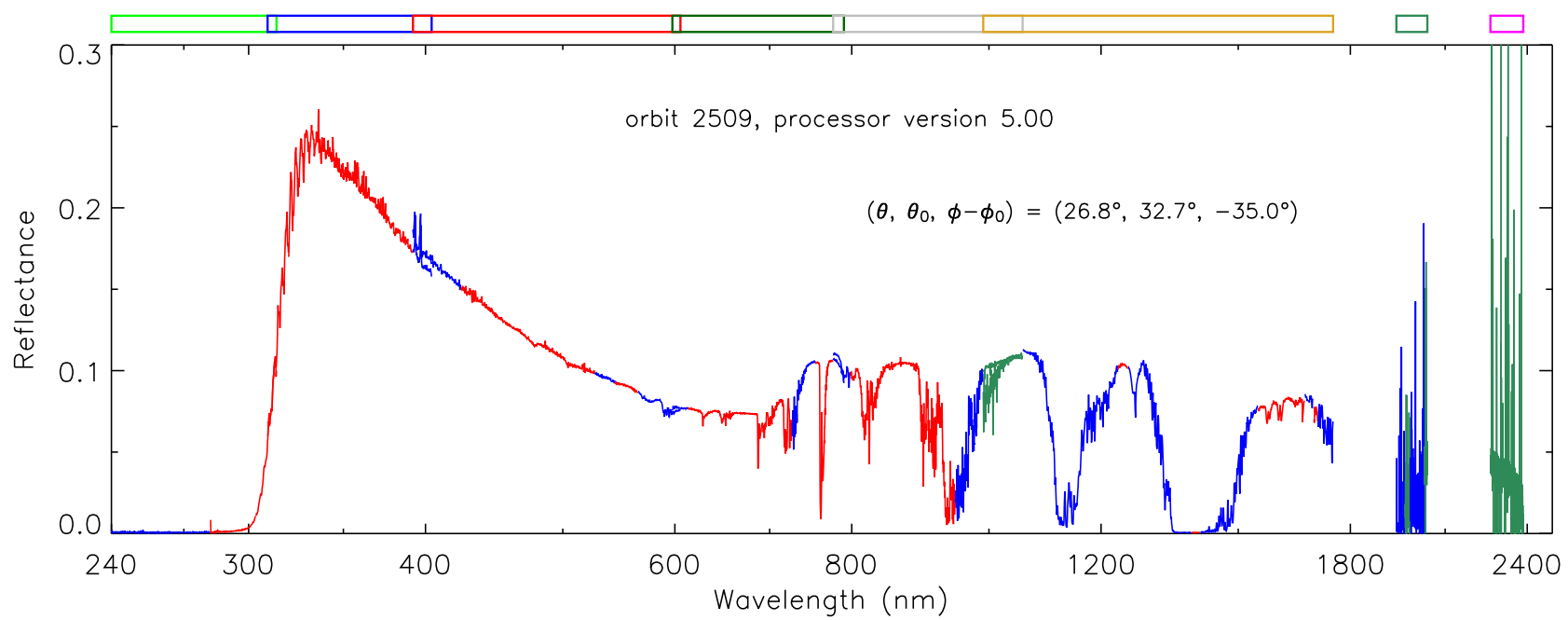

Fig. 2. Reflectance spectrum measured by SCIAMACHY, taken from verification orbit 2509, dated 23 August 2002. The coloured bars on top of the graph indicate the wavelength regions covered by the eight spectral channels, and their overlap, if any. The reflectance spectrum consists of red, green, and blue parts, which indicate clusters measured with an integration time of $0.25 \mathrm{~s}, 0.5 \mathrm{~s}$, and $1.0 \mathrm{~s}$. The spectrum represents the first (eastern) $1 \mathrm{~s}$ footprint of the upper state shown in Fig. 1.

constant $c_{\text {pol }}$, subject of this paper, performs a correction on the radiance for the effects of polarisation and can only be determined in-flight, as it is scene dependent. This is explained in detail in the next section. The solar spectrum does not need to be corrected for polarisation effects because sunlight is unpolarised.

\section{Polarisation correction}

Atmospheric radiation is usually described by means of a Stokes vector $\{I, Q, U, V\}$, in which the component $I$ refers to the radiance defined in the previous section, and the components $Q$ and $U$ both characterise the linear polarisation of the light (van de Hulst, 1981). Circular polarisation, described by the Stokes parameter $V$, is negligible for atmospheric radiation (Coulson, 1988) and never taken into account. The linear polarisation parameters $Q$ and $U$ are defined with respect to a reference plane, and are usually expressed in terms of the degree of linear polarisation $P$ and the direction of polarisation $\chi$ according to

$Q / I=P \cos 2 \chi$

$U / I=P \sin 2 \chi$.

The direction of polarisation $\chi$ is mainly determined by the geometry that defines the sun-atmosphere-satellite system. A recent study has shown that it deviates very little from its theoretical single scattering value (Schutgens et al., 2004). This means that we can use $\chi \approx \chi_{\text {ss }}$, where $\chi_{\text {ss }}$ is calculated from geometry only (e.g. Tilstra et al., 2003). The degree of polarisation $P$ does not only contain scattering geometry information, but also information about the properties of the observed scene. For wavelengths below $300 \mathrm{~nm}$, where ozone absorption is so strong that single scattering is a good approximation, the degree of polarisation only depends on the scattering geometry, and is given by

$P_{\mathrm{ss}}=\frac{1-\cos ^{2} \Theta}{1+\Delta+\cos ^{2} \Theta}$,

where $\Theta$ is the single scattering angle, and $\Delta$ is a correction factor for depolarisation due to molecular anisotropy. At $350 \mathrm{~nm}, \Delta=0.0621$ (Bates, 1984). Equation (6) is not valid above $300 \mathrm{~nm}$, but may serve as a practical upper limit, or first estimate, for polarisation validation (see e.g. Krijger et al., 2005).

Spectrometers like GOME or SCIAMACHY are unfortunately not only sensitive to the intensity of the radiation, but also to its polarisation. More specifically, the instrument's response to atmospheric light is given by (Slijkhuis, 2001)

$$
\begin{aligned}
R_{\mathrm{pol}} & =R_{\mathrm{unpol}} / c_{\mathrm{pol}} \\
& =\left(1+\mu_{2}^{D} Q / I+\mu_{3}^{D} U / I\right) \cdot R_{\mathrm{unpol}},
\end{aligned}
$$

where $R_{\text {pol }}$ stands for the reflectance including polarisation effects (i.e. as it would be reported by SCIAMACHY), and $R_{\text {unpol }}$ for the true reflectance. The constants $\mu_{2}^{D}$ and $\mu_{3}^{D}$ are the optical response functions of the spectral channels to $Q$ and $U$, respectively. They both depend on wavelength, spectral channel, and the position of the scan mirror. To retrieve the true reflectance $R_{\text {unpol }}$, Eq. (7) should be inverted, but this requires knowledge of the Stokes parameters $Q$ and $U$. For that reason, SCIAMACHY is equipped with seven PMDs which measure the polarisation with broad spectral bands centred at six wavelengths. A correction for the sensitivity to polarisation can then be applied using Eq. (7) (Slijkhuis, 2001). 


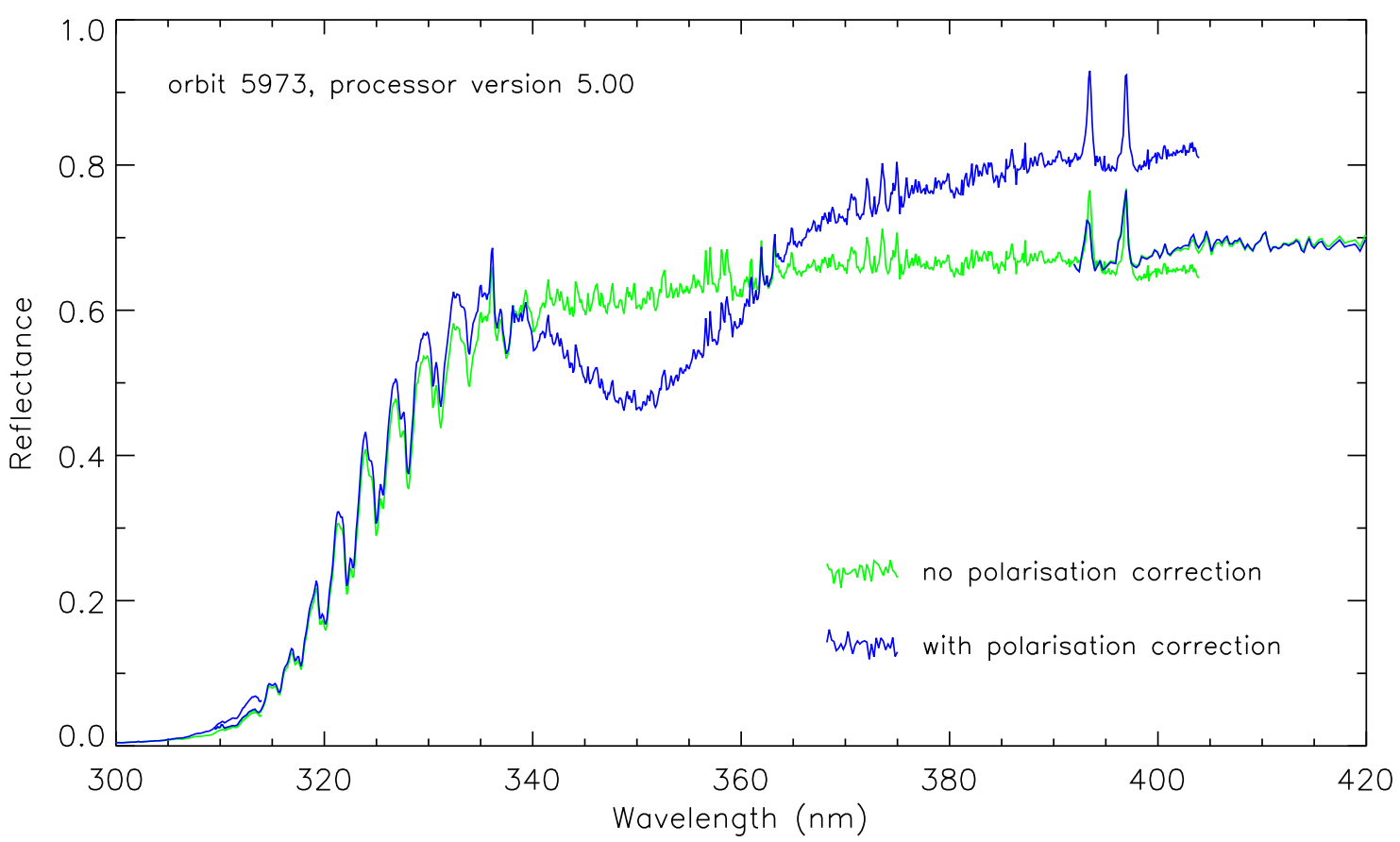

Fig. 3. Portion of a SCIAMACHY reflectance spectrum taken from a clouded scene over the Atlantic Ocean. The green spectrum is the reflectance before polarisation correction has been applied. Polarisation features are visible, but small, because the degree of polarisation is fairly low for this particular scene. The blue spectrum is the reflectance after applying polarisation correction. Obviously, the operational polarisation correction does not improve the spectrum.

At the moment, however, the quality of this polarisation correction is not sufficient, as is illustrated by Fig. 3. In this figure we have plotted (in green) part of a reflectance spectrum as measured by SCIAMACHY, with the polarisation correction not applied. The observed scene was completely clouded and located over the Atlantic Ocean. As clouds tend to decrease the degree of polarisation $P$, and hence $Q / I$ and $U / I$, the spectrum does not suffer much from the neglect of not applying the polarisation correction (cf. Eq. 7). When switching on polarisation correction, however, a strong polarisation feature is introduced in the reflectance spectrum (blue curve). In this case the polarisation feature is caused by the fact that the data processor is not functioning correctly.

However annoying, this polarisation feature in the original spectrum around $350 \mathrm{~nm}$ also has the potential to supply information about the polarisation of the detected radiation. In the next section the polarisation feature around $350 \mathrm{~nm}$ will be used to retrieve polarisation Stokes parameters from the reflectance spectrum measured by SCIAMACHY.

\section{Alternative polarisation retrieval algorithm}

Figure 4 explains the steps of the polarisation retrieval algorithm we will present in this section. In the first window, denoted by (a), we have plotted the reflectances of clusters 9 and 10 , as measured by SCIAMACHY, and without any correction for polarisation effects. Both clusters are part of spectral channel 2 (cluster 9: 320-392 nm; cluster 10: 309$320 \mathrm{~nm}$ ), and are, in this case, read out with an integration time (IT) of $0.25 \mathrm{~s}$. It was already explained in Sect. 3 that the reflectance $R_{\text {pol }}$ shown in window (a) is linked to the true reflectance $R_{\text {unpol }}$ according to

$R_{\mathrm{pol}}=(1+P \beta) R_{\mathrm{unpol}}$,

where

$\beta=\mu_{2}^{D} \cos 2 \chi+\mu_{3}^{D} \sin 2 \chi$.

In step (b) we take the response functions $\mu_{2}^{D}$ and $\mu_{3}^{D}$, which depend only on the position of the scan mirror, from the calibration database. Their specific shape reveals the origin of the polarisation feature at $350 \mathrm{~nm}$ seen in (a). Next, in step (c), we make the assumption that the direction of polarisation $\chi$ may be approximated by its single scattering value $\chi_{\mathrm{ss}}$ (Schutgens et al., 2004). Using $\chi=\chi_{\mathrm{ss}}$, we obtain $\beta_{\mathrm{ss}}$. Owing to the specific shape of $\beta_{\mathrm{ss}}$, we can determine two wavelengths $\lambda_{1}$ and $\lambda_{2}$ for which $\beta_{\mathrm{ss}}=0$. These are, as can be seen from Eq. (8), wavelengths for which the reflectance signal measured by SCIAMACHY is insensitive to the degree of polarisation $P$, and therefore unaffected by the polarisation sensitivity of the spectral channel. The wavelengths $\lambda_{1}$ and $\lambda_{2}$ are determined for every spectrum. Typical values are $\lambda_{1}=335 \pm 5 \mathrm{~nm}$ and $\lambda_{2}=365 \pm 5 \mathrm{~nm}$. 

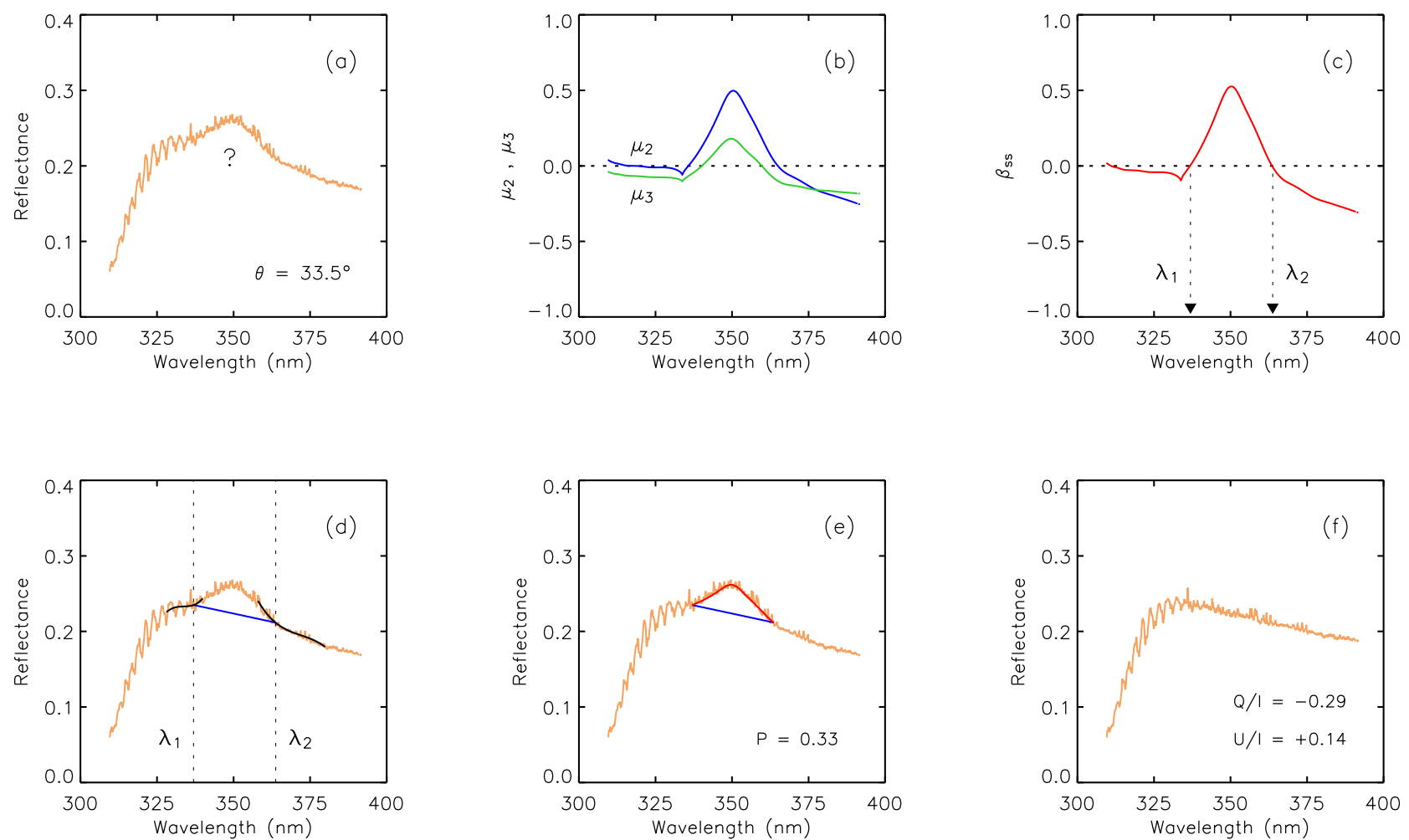

Fig. 4. Graphical description of the steps of the polarisation retrieval algorithm. (a) Work with the reflectance not corrected for polarisation effects. (b) Determine the polarisation sensitivity elements $\mu_{2}$ and $\mu_{3}$ of the spectral channel. (c) Calculate $\beta_{\mathrm{ss}}$, and determine the two wavelengths $\lambda_{1}$ and $\lambda_{2}$ for which the reflectance is "insensitive" to polarisation $\left(\beta_{\mathrm{ss}} \approx 0\right)$. (d) Construct a linear function between $\lambda_{1}$ and $\lambda_{2}$.

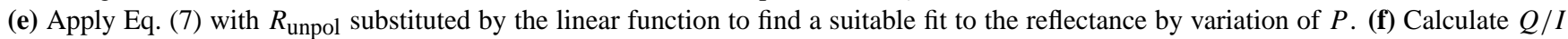
and $U / I$ and correct for polarisation using Eq. (7). The polarisation feature around $350 \mathrm{~nm}$ is successfully removed from the reflectance.

Now, step (d) is based on the essential assumption that the shape of the Earth's reflectance spectrum between these two wavelengths is linear. As the two wavelengths are no more than $\sim 30 \mathrm{~nm}$ apart this is in fact quite a reasonable assumption which has been checked with validated GOME spectra. So we determine the linear function between $\lambda_{1}$ and $\lambda_{2}$. In step (e) we substitute $R_{\text {unpol }}$ in Eq. (8) for this linear function, and fit the resulting equation to the reflectance $R_{\mathrm{pol}}$ shown in (a) by variation of $P$, which we assume to be constant over this small wavelength range. Step (f), finally, involves calculating the Stokes parameters $Q / I$ and $U / I$ from Eqs. (4) and (5), and finding the true reflectance $R_{\text {unpol }}$ by inversion of Eq. (8).

The six-step algorithm described above is simple and robust. The only real approximation made is the assumption that the reflectance changes linearly over the wavelength interval $\left(\lambda_{1}, \lambda_{2}\right)$. Deviations caused by this approximation should not be large and we estimate them to be of the order of a few percent for worst-case scenarios only. Secondly, we have assumed $\chi=\chi_{\text {ss }}$ (Schutgens et al., 2004). Note that the current SCIAMACHY data processor is built around the same simplification. In special situations, like exact backscattering, this may cause noticeable deviations. Thirdly, it is assumed that the degree of polarisation is constant over the interval $\left(\lambda_{1}, \lambda_{2}\right)$. The retrieved $P$ should therefore be considered as an effective $P$ over the full "bandwidth" of the polarisation feature. The assumption that $P$ can be considered spectrally constant is implemented in the data processor as well (Slijkhuis, 2001), and here the relevant wavelength range is even much larger, $67 \mathrm{~nm}$ to be exact. Last but not least we have assumed that the response functions $\mu_{2}^{D}$ and $\mu_{3}^{D}$ are known correctly. The latter assumption is not trivial in the case of SCIAMACHY, which currently has calibration errors.

The main advantage of the retrieval algorithm, apart from its simple implementation, is the fact that it does not require input from the PMDs. The operational processor requires both the spectral channel and the PMD signals to calculate polarisation. So, the operational algorithm depends on much more calibration key data than the alternative algorithm. Notice that an absolute calibration of the reflectance is unimportant for the alternative polarisation retrieval, and that absolute calibration errors in $\mu_{2}^{D}$ and $\mu_{3}^{D}$ would lead to wrong $Q / I$ and $U / I$, but their combination would still result in a right polarisation correction of the reflectance, and removal of the polarisation feature at $350 \mathrm{~nm}$. 

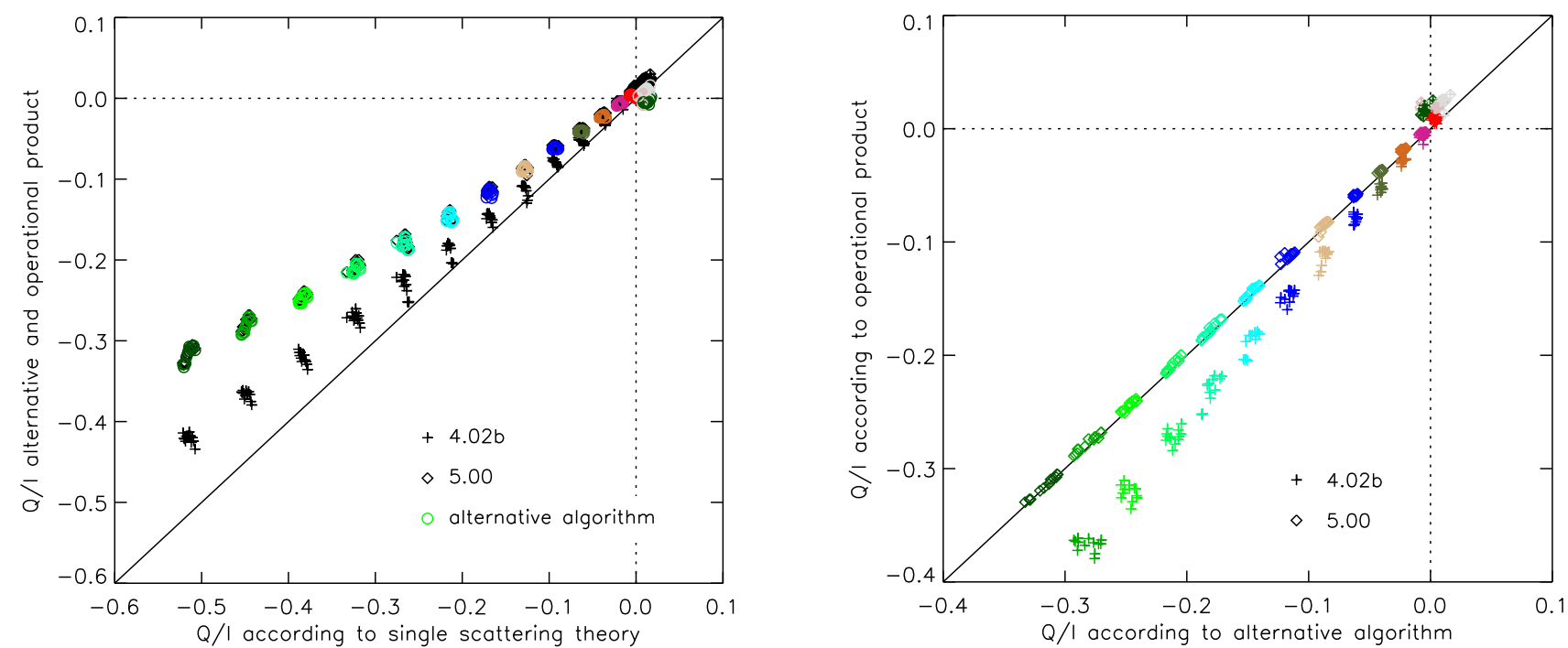

Fig. 5. Left window: retrieved normalised Stokes parameter $Q / I$ versus the theoretical single scattering value for (i) the operational product, software version $4.02 \mathrm{~b}$, (ii) the operational product, software version 5.00, and (iii) our alternative algorithm. The data are from a cloud-free state over the Sahara, the lower state shown in Fig. 1. Colours, where used, are used to distinguish between different nadir viewing angles. Left and right correspond to east and west. Right window: we have plotted the same results, but now (i) and (ii) are plotted against (iii).

At this point it should be mentioned that a similar method as the one presented in this paper, based on the same ideas and assumptions, has recently been published by McLinden et al. (2004). Here the degree of linear polarisation is derived from limb-scattered sunlight measured by the OSIRIS instrument (flown on the Odin satellite). The polarisation retrieval is again realised by making use of the instrumental sensitivity to the state of polarisation of the detected light.

In the next section we will focus on the Stokes parameters, and compare these with the ones obtained by the operational product as well as with model calculations.

\section{Results}

\subsection{Alternative algorithm versus operational product}

For a first verification we focus on the Sahara state shown in Fig. 1, which was completely cloud-free at the time of SCIAMACHY's overpass. Around these latitudes, spectral channel 2 has an integration time of only $0.25 \mathrm{~s}$ which results in many small pixels. In Fig. 5 we compare for all these pixels the operational product with our own polarisation retrieval algorithm. In the left window we plotted the normalised Stokes parameter $Q / I$ as a function of the theoretical single scattering value for three cases. The first two cases are the $Q / I$ of the operational product, for processor versions $4.02 \mathrm{~b}$ and 5.00. These are indicated by black plusses and diamonds, respectively. The coloured circles are the $Q / I$ obtained using our alternative polarisation retrieval algorithm.

The colours enable the reader to distinguish between different scan mirror positions. Points having the same colour relate to virtually the same scattering geometry, and therefore show almost identical values for the single scattering $Q / I$. For cloud-free scenes the scattering geometry is the most important parameter determining the polarisation of reflected sunlight, which explains why colours are also grouped together along the vertical axis of Fig. 5. Notice that the single scattering $Q / I$ is a valid approximation for wavelengths below $300 \mathrm{~nm}$ (Schutgens and Stammes, 2002, 2003) so that the left window of Fig. 5 basically compares $Q / I$ at and below $300 \mathrm{~nm}$ with $Q / I$ at $350 \mathrm{~nm}$.

All three approaches in the left window roughly follow the behaviour of the theoretical single scattering result, which is promising. However, there is disagreement about the absolute magnitude of the polarisation. Software version 5.00 is in agreement with the alternative polarisation retrieval algorithm, but software version $4.02 \mathrm{~b}$ is not. This is shown more clearly in the right window of Fig. 5. Here we have plotted $Q / I$ of the operational product against the $Q / I$ found by the polarisation retrieval of this paper. Plots for the Stokes parameter $U / I$ turn out to be very similar to those in Fig. 5, which is why these are not presented.

Next, in Fig. 6, we present a similar comparison, but now for a different nadir state of orbit 2509, where SCIAMACHY observed a less homogeneous part of the Sahara desert, which was partly clouded. The left window is interesting, because it clearly illustrates the depolarising effect of clouds. More importantly for this paper, this time there is good agreement between all three algorithms (see right window of Fig. 6). Especially the sudden agreement between the two operational products is striking. For the entire orbit, software version 5.00 systematically behaves better than 

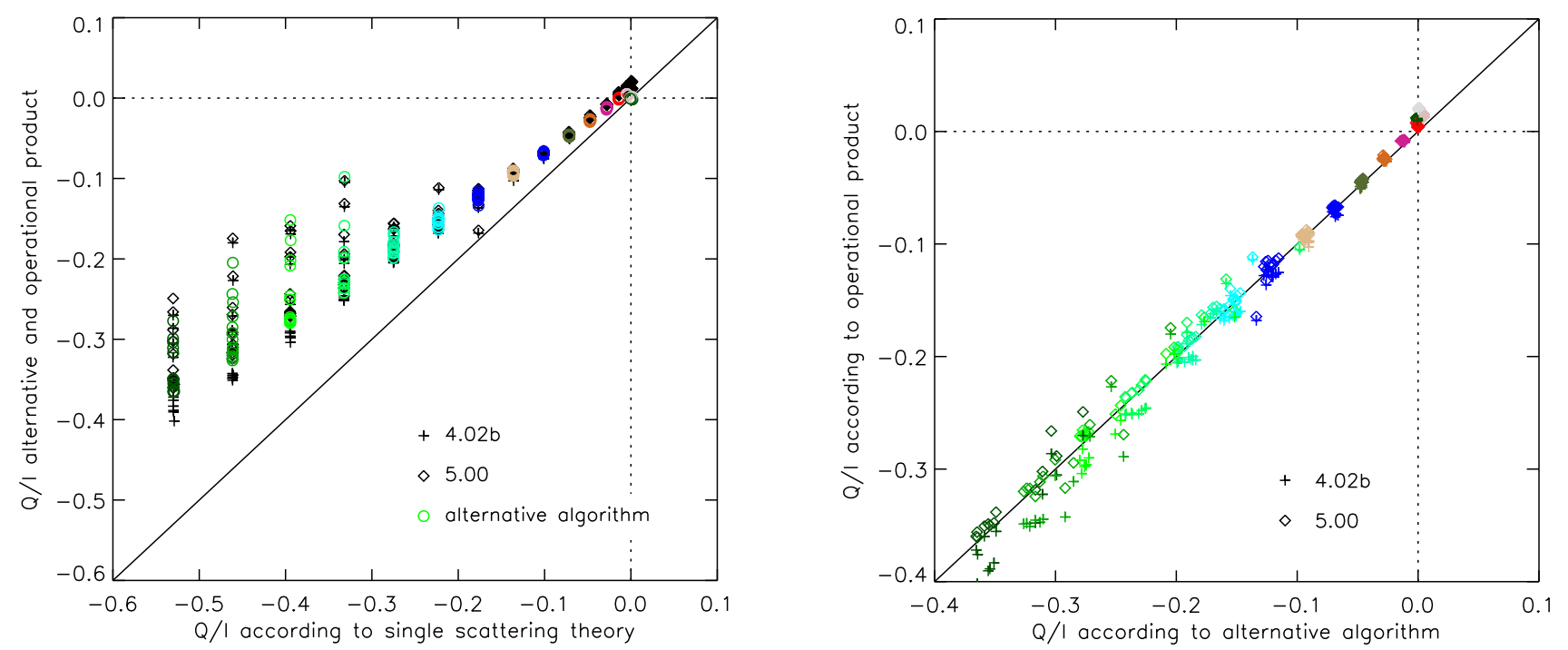

Fig. 6. Same as Fig. 5, but now for a different state of orbit 2509 over the Sahara desert. This state is partially clouded which results in a higher depolarisation (the cloud is located in right lower corner of the state). Notice the difference w.r.t. Fig. 5, where there was only agreement between the alternative algorithm and software version 5.00 of the operational product. Now all three algorithms agree about the value of $Q / I$. The same is true for $U / I$ (not shown).

software version 4.02b. Agreement between our own algorithm and software version 5.00 of the operational product is as good as sketched in Figs. 5 and 6 at latitudes $30^{\circ} \mathrm{S}-60^{\circ} \mathrm{N}$, but becomes worse near the polar regions. It should be noted that we only looked at verification orbits 2509 and 2510 .

Also note that the agreement with the official SCIAMACHY polarisation product only "suggests" that our alternative polarisation retrieval is working properly. It is not a proof. Obviously, we require another verification approach.

\subsection{Comparison with an analytical polarisation model}

Needing a second verification tool, we make use of the model introduced by Tilstra et al. (2003). In this simple model, atmospheric polarisation is the result of a Rayleigh scattering atmosphere including only single scattering. Surface reflection is included by adding a depolarising Lambertian surface below the atmosphere. Clouds are not included in the model, so the model can only be used for cloud-free scenes. In this model, the degree of polarisation $P_{\text {model }}$ is given by

$P_{\text {model }}=\frac{1-\cos ^{2} \Theta}{1+\Delta+\gamma+\cos ^{2} \Theta}$,

where $\Theta$ is the single scattering angle, and

$\gamma=\frac{4}{3} \frac{A M}{\Delta^{\prime}}\left\{\frac{\exp \left(-M \tau_{R}\right)}{1-\exp \left(-M \tau_{R}\right)}\right\}$.

In these equations, $M=1 / \mu+1 / \mu_{0}$ is the geometrical airmass factor, and $\Delta=2 \rho_{\mathrm{n}} /\left(1-\rho_{\mathrm{n}}\right)$ is a correction factor for depolarisation due to molecular anisotropy, as is $\Delta^{\prime}=\left(1-\rho_{\mathrm{n}}\right) /\left(1+\rho_{\mathrm{n}} / 2\right)$, where $\rho_{\mathrm{n}}$ is the depolarisation factor of air. At $350 \mathrm{~nm}, \rho_{\mathrm{n}}=0.0301$, and we find $\Delta=0.0621$ and $\Delta^{\prime}=0.9555$ (Bates, 1984). The other variables, $A$ and $\tau_{R}$, are the surface albedo and the Rayleigh optical thickness of the atmosphere. Surface reflection is treated by this analytical model as just another depolarisation correction factor (cf. Eq. 6). For the surface albedo $A$ and the Rayleigh optical thickness $\tau_{R}$ we use reasonable values $\left(A=0.3 ; \tau_{R}=0.6\right)$. However, both, and the surface albedo in particular, are not meant to reproduce the actual values, but also account for multiple scattering and other matters that are not or insufficiently treated by the model.

The result of the comparison is shown in Fig. 7. Here we have plotted the degree of polarisation $P$, as found by our own polarisation retrieval for the same state as shown in Fig. 6, as a function of the scattering angle $\Theta$. The meaning of the colours used is the same as before. The black diamonds are the model results calculated using Eqs. (10) and (11). Here $A$ and $\tau_{R}$ were kept fixed, i.e. all the black diamonds in Fig. 7 have the same $A$ and $\tau_{R}$.

Apparently the model is capable of explaining the dependence of $P$ on $\Theta$. Below $145^{\circ}$ the measured data spread out which is caused by the fact that a cloud is present in part of the state. Clouds generally lower the degree of polarisation of atmospheric radiation. The model does not take clouds into consideration, which in this case is not a real problem because below $145^{\circ}$ some unclouded pixels are also present. For these pixels, the model data mimic the measurement data in great detail. The conclusion can be drawn that the alternative polarisation algorithm responds properly to scene characteristics as well as to scattering geometry. On the other hand, it cannot be fully excluded that an overall multiplicative error in $P$ would exist. 


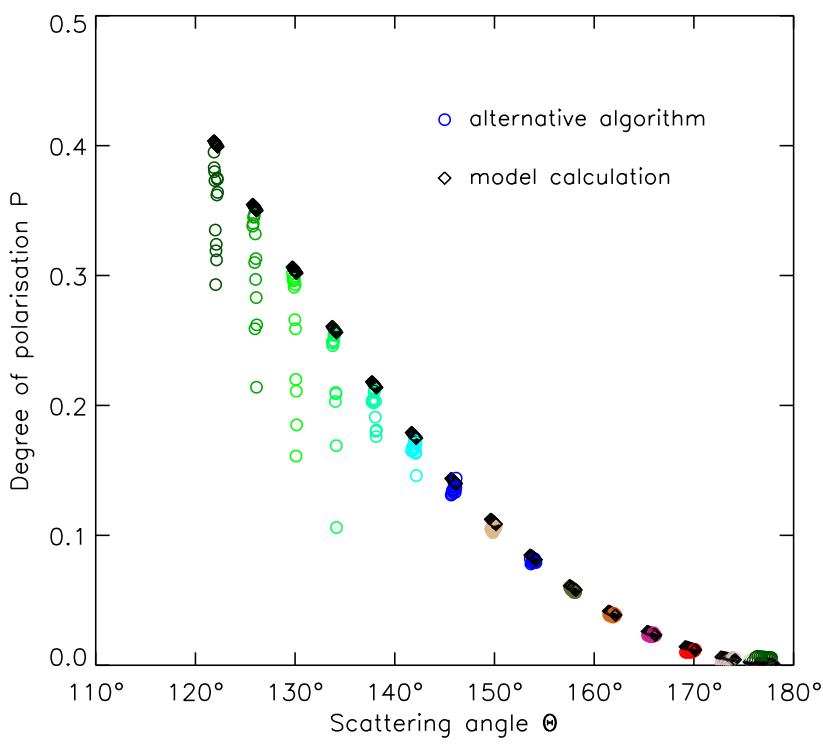

Fig. 7. Degree of linear polarisation $P$ versus the single scattering angle $\Theta$ for the same state shown in Fig. 6. The coloured circles are produced by the alternative polarisation retrieval of this paper, the black diamonds arise from a single scattering model that includes surface reflection below a cloud-free atmosphere. The pixels that so nicely follow the model calculations are cloud-free, those that deviate are clouded. Thus, there is a very high correlation between the actual scene and the results of the polarisation retrieval algorithm.

\subsection{Integration times}

The SCIAMACHY operational product provides normalised Stokes parameters $Q / I$ and $U / I$ for all the integration times that are used in a particular state. The polarisation retrieval discussed in this paper basically only calculates the polarisation for the IT with which the measurements of cluster 9 (320-392 nm) were performed. However, polarisation values for longer ITs may be obtained by binning of the reflectances of the small pixels. For example, the IT of the two specific nadir states discussed in this paper is $0.25 \mathrm{~s}$. By simply binning the reflectances of the $0.25 \mathrm{~s}$ pixels (cf. Fig. 1) we can build the reflectance that would have been measured had the IT been longer. This reflectance then goes into the algorithm discussed in Sect. 4 to arrive at polarisation values for $0.5 \mathrm{~s}$, $1 \mathrm{~s}, 5 \mathrm{~s}$, or even $10 \mathrm{~s}$ IT. The quality of these polarisation values is by definition just as high if not better.

\section{Conclusions}

In this paper we have introduced an alternative method for the retrieval of polarisation in the UV by the satellite instrument SCIAMACHY. The method does not rely on the input of the relevant Polarisation Measurement Device in the UV (PMD 1) but instead uses only the reflectance signal (not corrected for polarisation effects). This makes the al- ternative algorithm more robust and less susceptible to calibration errors than the operational SCIAMACHY polarisation retrieval. Furthermore, the alternative algorithm automatically corrects for the polarisation-sensitive feature in the SCIAMACHY spectra around $350 \mathrm{~nm}$.

Using this alternative polarisation retrieval, we were able to validate the polarisation Stokes parameters $Q / I$ and $U / I$ of the two latest versions of the SCIAMACHY data product (at the time of writing: software versions $4.02 \mathrm{~b}$ and 5.00). As it turns out, the overall behaviour of the newer version 5.00 of the operational product is realistic, and distinctly better than version 4.02 , but still often both software versions lack the proper values of $Q / I$ and $U / I$. Without further analysis, we cannot say whether it is the calibration of the operational data processor that is insufficient or the data processor itself that is working incorrectly (including the calibration step from level-1b to level-1c by the SciaL1C tool).

To verify the alternative algorithm we use an analytical model to mimic the retrieved degree of polarisation for different viewing geometries. The model is able to follow the angular behaviour related to different scattering geometries very closely, indicating that the alternative algorithm is able to cope with the changing conditions in a proper manner. This can also be concluded from the proper response to clouds when present in an observed scene.

Acknowledgements. The work presented in this paper was financed by the Netherlands Agency for Aerospace Programmes (NIVR) and the Space Research Organisation Netherlands (SRON) through project EO-076. We would like to thank the European Space Agency (ESA) and the Deutsches Zentrum für Luft- und Raumfahrt (DLR) for providing the SCIAMACHY data, and J. Frerick from ESA in particular for supplying us with the latest versions of verification orbits 2509 and 2510 .

Edited by: U. Platt

\section{References}

Bates, D. R.: Rayleigh scattering by air, Planet, Space Sci., 32, 785-790, 1984.

Bovensmann, H., Burrows, J. P., Buchwitz, M., Frerick, J., Noël, S., Rozanov, V. V., Chance, K. V., and Goede, A. P. H.: SCIAMACHY: Mission objectives and measurement modes, J. Atmos. Sci., 56, 127-150, 1999.

Burrows, J. P., Weber, M., Buchwitz, M., Rozanov, V., LadstätterWeißenmayer, A., Richter, A., de Beek, R., Hoogen, R., Bramstedt, K., Eichman, K.-U., Eisinger, M., and Perner, D.: The Global Ozone Monitoring Experiment (GOME): Mission concept and first scientific results, J. Atmos. Sci., 56, 151-175, 1999.

Callies, J., Corpacciolli, E., Eisinger, M., Hahne, A., and Lefebvre, A.: GOME-2 - Metop's second-generation sensor for operational ozone-monitoring, ESA Bull., 102, 28-36, 2000.

Coulson, K. L.: Polarization and Intensity of Light in the Atmosphere, A. Deepak, Hampton, Va, 1988.

Krijger, J. M., Tanzi C. P., Aben, I., and Paul, F.: Validation of GOME polarization measurements by method 
of limiting atmospheres, J. Geophys. Res., 110, D07305, doi:10.1029/2004JD005184, 2005.

McLinden, C. A., Haley, C. S., and Llewellyn, E. J.: Derivation of polarization from Odin/OSIRIS limb spectra, Geophys. Res. Lett., 31, L20112, doi:10.1029/2004GL020825, 2004.

Schutgens, N. A. J. and Stammes, P.: Parametrisation of Earth's polarisation spectrum in the ultra-violet, J. Quant. Spectrosc. Radiat., 75, 239-255, 2002.

Schutgens, N. A. J. and Stammes, P.: A novel approach to the polarization correction of spaceborne spectrometers, J. Geophys. Res., 108, D7, 4229, doi:10.1029/2002JD002736, 2003.

Schutgens, N. A. J., Tilstra, L. G., Stammes, P., and Bréon, F.-M.: On the relationship between Stokes parameters $Q$ and $U$ of atmospheric ultraviolet/visible/near-infrared radiation, J. Geophys. Res., 109, D09205, doi:10.1029/2003JD004081, 2004.
Slijkhuis, S.: SCIAMACHY level 0 to $1 \mathrm{c}$ processing algorithm theoretical basis document, Tech. Note ENV-ATB-DLR-SCIA0041, Dtsch. Zent. für Luft- und Raumfahrt, Oberpfaffenhofen, Germany, 2001.

Tilstra, L. G., Schutgens, N. A. J., and Stammes, P.: Analytical calculation of Stokes parameters Q and $\mathrm{U}$ of atmospheric radiation, Sci. Rep. WR 2003-01, Koninklijk Ned. Meteorol. Inst., De Bilt, Netherlands, 2003.

van de Hulst, H. C.: Light Scattering by Small Particles, Dover, Mineola, N. Y., 1981. 